\title{
Hadron Production Measurements for Neutrino Experiments by the NA61/SHINE Experiment at CERN
}

\author{
Alessandro Bravar* on behalf of the NA61 Collaboration \\ Universtiy of Geneva, Geneva, Switzerland \\ E-mail: Alessandro.Bravar@unige.ch
}

\begin{abstract}
As neutrino long baseline experiments enter a new domain of precision, important systematic errors due to poor knowledge of production cross sections for pions and kaons require more precise measurements. Among other goals, the NA61/SHINE (SHINE = SPS Heavy Ion and Neutrino Experiment) experiment at the CERN SPS aims at precision hadron production measurements (5\% and below) to improve the prediction of the neutrino flux for the T2K experiment at J-PARC. The spectrometer is equipped with a large set of TPCs, whose acceptance covers the relevant phase-space for the T2K beamline. Extensive $\mathrm{dE} / \mathrm{dx}$ measurements in the TPC, complemented by an upgraded Time-of-Flight system, provide particle identification over the whole kinematic range. These measurements are performed using a $30 \mathrm{GeV}$ proton beam impinging on carbon targets of different lengths, including a replica of the T2K target. Data were taken in 2007 and in 2009 after a major readout upgrade. Preliminary spectra for positive and negative pions from the 2007 run obtained with a $2 \mathrm{~cm}$ thick carbon target are presented.
\end{abstract}

35th International Conference of High Energy Physics - ICHEP2010,

July 22-28, 2010

Paris France

* Speaker. 


\section{Introduction}

In a long baseline neutrino oscillation experiment like T2K [1], neutrino oscillations are probed by comparing the neutrino flux measured at a detector far (Super-Kamiokande in this case) from the neutrino source (far means $L / E \gg 1$, where $L$ is the distance from the source and $E$ the neutrino energy) and comparing it with a prediction based on the neutrino flux unmodified by oscillations. The unoscillated flux is predicted on the basis of the flux measured at a near detector before neutrinos possibly oscillate and extrapolated to the far detector location with the help of a simulation of the neutrino beamline, which depends also on the production cross sections for pions and kaons which decay into neutrinos. As the intensity of neutrino beams produced at accelerators increases, the systematic uncertainties due to the poor characterization of the neutrino beam flux become a limiting factor for high precision neutrino oscillation experiments. To fully understand the neutrino beam, details of the production mechanisms of the beam have to be known and understood. The uncertainties in the production of $\pi^{+}$and $\mathrm{K}^{+}$mesons lead to important uncertainties in the oscillation analysis. Furthermore, they preclude precise measurements of neutrino cross sections.

T2K [1] is a long baseline neutrino oscillation experiment in Japan, which uses a high intensity neutrino source produced at J-PARC. Neutrinos are created in the decays of $\pi^{+}$and $\mathrm{K}^{+}$mesons produced by a high intensity $30 \mathrm{GeV}$ proton beam impinging on a $90 \mathrm{~cm}$ long graphite rod. Since there are no hadron production data for $\mathrm{p}+\mathrm{C}$ interactions at $30 \mathrm{GeV}$ and extrapolations from existing measurements are not always reliable, a precise measurement of $\pi^{+}$and $\mathrm{K}^{+}$spectra produced off the T2K target is mandatory. A detector with a very large angular acceptance and good particle identification capabilities over a broad momentum range, like [2], is needed.

The NA61/SHINE experiment (SHINE = SPS Heavy Ion and Neutrino Experiment) [2] is a large acceptance hadron spectrometer at the CERN-SPS for the study of the hadronic final states produced in interactions of various beam particles (protons, $\pi$ 's, and heavy ions) with a variety of fixed targets and incident beam energies in the range of SPS energies (from $\sim 10 \mathrm{GeV}$ to $\sim 350 \mathrm{GeV}$ ). NA61 aims at precision hadron production measurements (5\% and below) to improve the prediction of the neutrino flux for the T2K experiment at J-PARC using a $30 \mathrm{GeV}$ proton beam and carbon targets of different lengths, including a replica of the T2K target. For an overview of the NA61 physics program see presentations of M. Unger [3] and P. Staszel [4] at this conference.

Recently, a series of dedicated hadron production experiments, such as HARP [5] at the CERN-PS and MIPP [6] at Fermilab, have been carried out to address similar issues. Precise measurements of pion and kaon production in $\mathrm{p}+\mathrm{p}$ interactions at $158 \mathrm{GeV} / \mathrm{c}$ are available from NA49 [7]. NA49 measured also pion production in p+C interactions at the same energy [8].

\section{The NA61/SHINE Detector}

The set-up of NA61/SHINE is shown in Fig. 1. The main components of the detector were constructed and used by the NA49 experiment [9]. Charged particle trajectories are measured in four large volume Time Projection Chambers (TPCs). Two TPCs (VTPC-1 and VTPC-2), are located inside two super-conducting dipole magnets with a maximum bending power of $9 \mathrm{Tm}$. In order to detect low momentum particles produced at large polar angles a much weaker magnetic field of $\sim 1.1 \mathrm{Tm}$ was used. Two large TPCs with a volume of $25 \mathrm{~m}^{3}$ each (MTPC-L and MTPC- 


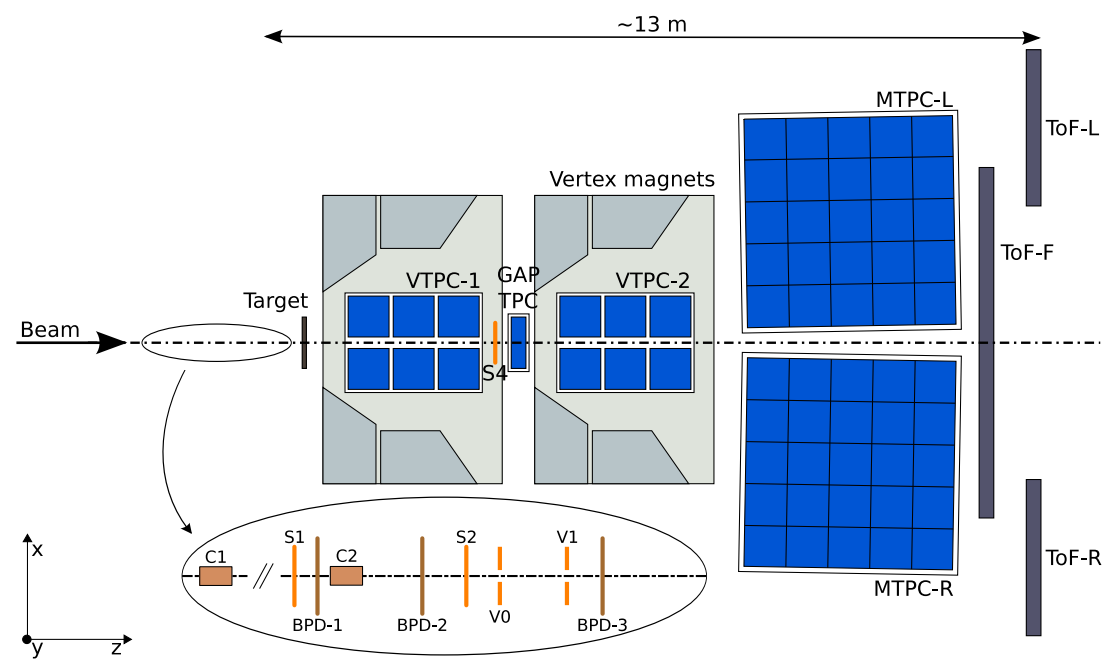

Figure 1: The layout of the NA61/SHINE detector (top view, not to scale). The inset shows the beamline detectors used to measure and select incoming beam particles.

$\mathrm{R})$, are positioned downstream of the magnets symmetrically with respect to the beam line. The incident beam particles are measured with three proportional chambers (BPDs) and identified by two beamline Cherenkov counters.

The apparatus is supplemented by Time of Flight (ToF) detectors located downstream of the main TPCs. In order to extend the acceptance for pion and kaon identification, a new forward ToF detector (ToF-F) was built as required for the T2K measurements. Although the ToF-F is located in the forward region, closing the gap between the ToF-R and ToF-L walls, it was primarily conceived to measure low momentum particles of a few $\mathrm{GeV} / c$ produced at large polar angles $(\theta>50 \mathrm{mrad})$, which are bent back into the detector acceptance by the vertex magnets. The resolution of the new ToF-F wall is $\approx 110 \mathrm{ps}$, providing a $5 \sigma \pi / \mathrm{K}$ separation at $3 \mathrm{GeV} / \mathrm{c}$ (see Fig. 2 left). The ToF-F provides full acceptance coverage of the $\mathrm{T} 2 \mathrm{~K}$ beamline phase-space.

\section{Data Analysis}

Critical for this analysis is the identification of the particles produced in $\mathrm{p}+\mathrm{C}$ interactions. Depending on the momentum range, different approaches have been adopted, which lead also to different event and track selection criteria. Figure 2 shows the Particle Identification (PID) performance of the NA61 detector based on ToF measurements (left) and specific energy loss, $\mathrm{dE} / \mathrm{dx}$, measurements in the TPCs. By combining the ToF with the $\mathrm{dE} / \mathrm{dx}$ measurements high purity $\pi / \mathrm{K} / \mathrm{p}$ samples, close to $100 \%$, can be obtained over the whole momentum range as illustrated in Fig. 3. Moreover in the $1-4 \mathrm{GeV} / c$ momentum region PID is only possible using the ToF method.

Three independent analysis methods have been thus developed:

1) analysis of $\pi^{-}$mesons via measurements of negatively charged particles with no PID [10]; this analysis is motivated by the observation that most of primary negatively charged particles are indeed $\pi^{-}$, and therefore can be performed without particle identification,

2) analysis of $\pi^{+}$and $\pi^{-}$mesons identified via the $\mathrm{dE} / \mathrm{dx}$ method alone for low momenta below $1 \mathrm{GeV} / c$ [11], and 

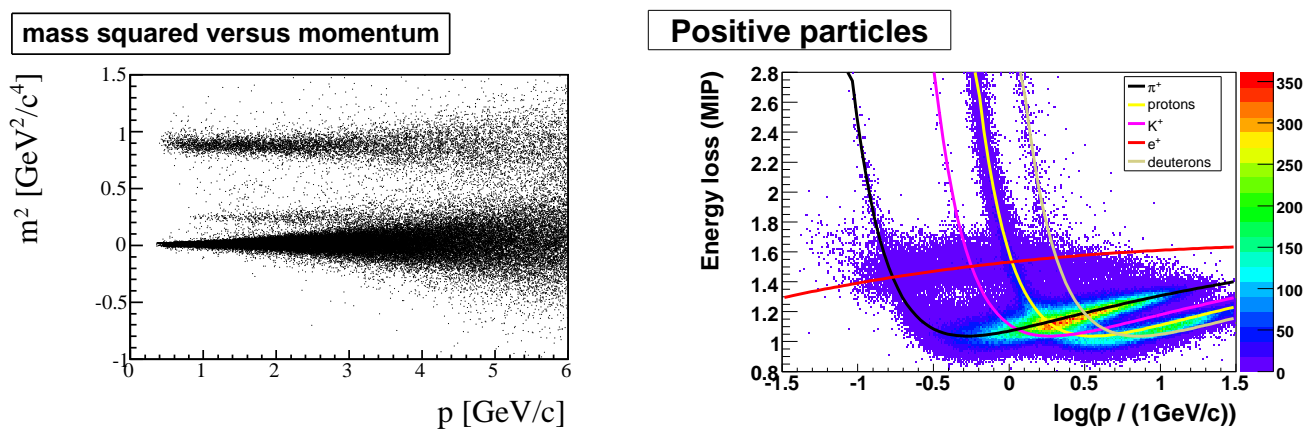

Figure 2: Particle Identification performance of the NA61 detector. Left: $m^{2}$ vs. momentum evaluated from ToF measurements. Right: specific energy loss, dE/dx, measurements in the TPCs.
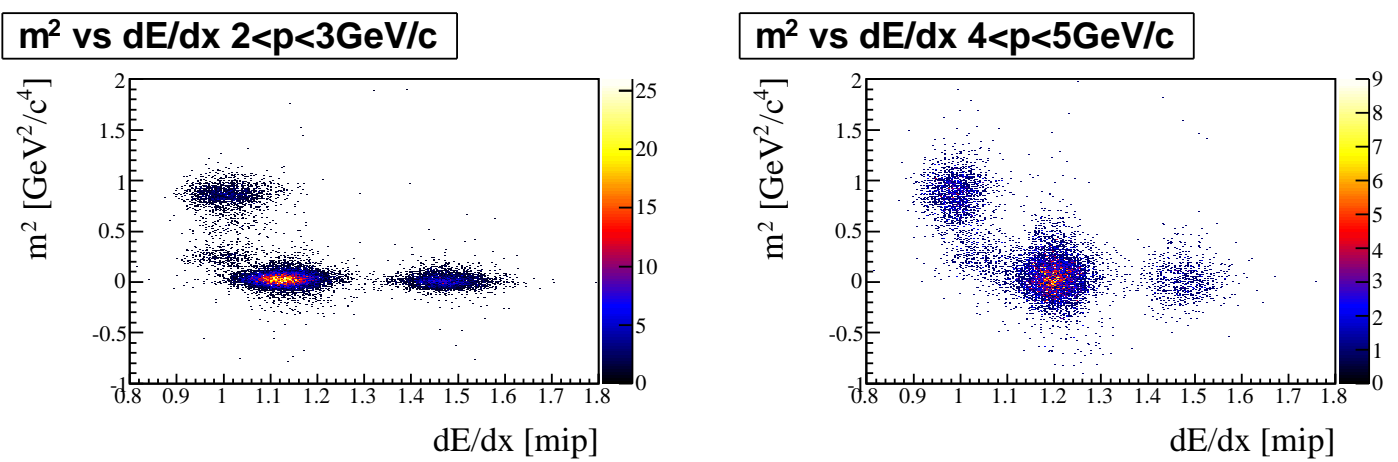

Figure 3: Examples of two-dimensional $\mathrm{m}^{2}-\mathrm{dE} / \mathrm{dx}$ distributions. 4 islands (left to right) corresponding to protons, kaons, pions, and electrons can be clearly identified.

3) analysis of $\pi^{+}$and $\pi^{-}$mesons identified via the combined ToF-dE/dx PID method for momenta above $1 \mathrm{GeV} / c$ [12].

Each analysis yields fully corrected pion spectra with independently calculated statistical and systematical errors.

\section{First Pion Cross Section Results}

Figures 4 and 5 show preliminary $\pi^{+}$and $\pi^{-}$inclusive cross sections spectra obtained in $\mathrm{p}+\mathrm{C}$ interactions at $30 \mathrm{GeV}$ from the data collected in 2007 with a $2 \mathrm{~cm}$ thick graphite target. The different analyses, where they overlap, can be compared, as well. Systematical uncertainties are estimated to be below $20 \%$. These results will be soon finalized.

A ten times larger data set has been collected in 2009. This larger data sample will allow us to study also kaon production over a similar kinematic range. Additional measurements with the T2K replica target have been performed in 2010 . The replica target data are being analyzed and results will be soon available.

\section{References}

[1] Y. Itow et al., The JHF-Kamiokande neutrino project, arXiv:hep-ex/0106019 (2001).

[2] NA61 proposal, N. Antoniou et al., CERN-SPSC-2006-034, 2007-004, 2007-019. 

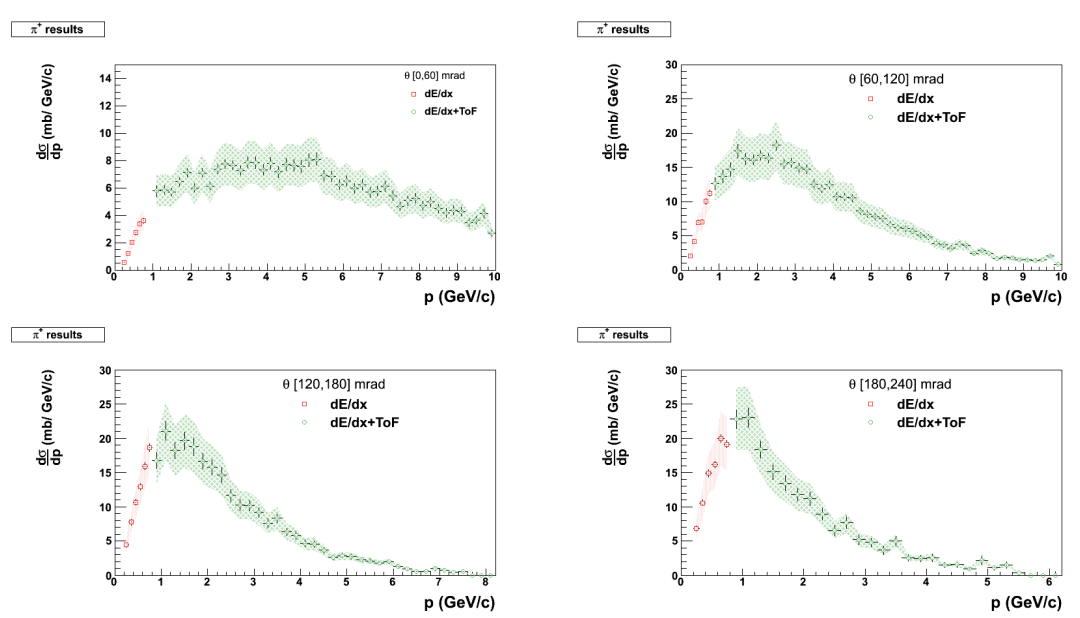

Figure 4: Preliminary differential cross section results for $\pi^{+}$production in $\mathrm{p}+\mathrm{C}$ interactions at $30 \mathrm{GeV}$. The spectra are shown in 4 bins of the polar angle $\theta$ between 0 and $240 \mathrm{mrad}$. The band represents the systematical error, which is estimated to be below $20 \%$. Results from different analyses are compared.
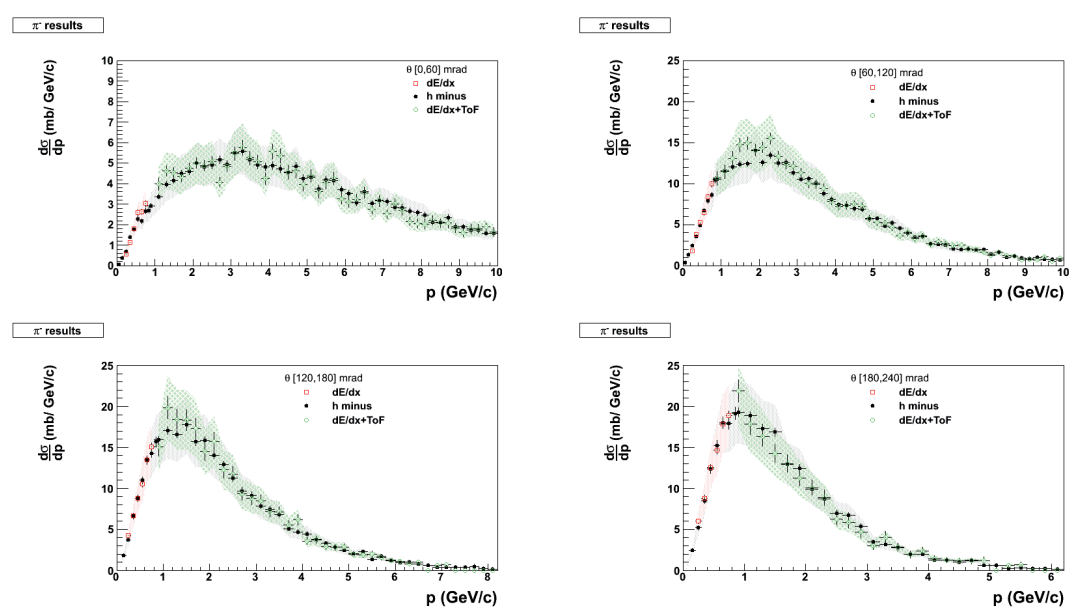

Figure 5: Preliminary differential cross section results for $\pi^{-}$production in $\mathrm{p}+\mathrm{C}$ interactions at $30 \mathrm{GeV}$.

[3] M. Unger [NA61 Collaboration], these proceedings.

[4] P. Staszel [NA61 Collaboration], these proceedings.

[5] M. Apollonio et al. [HARP Collaboration], Phys. Rev. C80 (2009) 035208.

[6] J. Paley [MIPP Collaboration], AIP Conf. Proc. 981 (2008) 154.

[7] C. Alt et al. [NA49 Collaboration], Eur. Phys. J. 45 (2006) 343;

T. Anticic et al. [NA49 Collaboration], arXiv:hep-ex/1004.1891 (2010).

[8] C. Alt et al. [NA49 Collaboration], Eur. Phys. J. 49 (2007) 897.

[9] S. Afanasev et al. [NA49 Collaboration], Nucl. Inst. Meth. A 430 (1999) 210.

[10] T. Palczewski, Ph.D. thesis in preparation.

[11] M. Posiadala, Ph.D. thesis in preparation.

[12] S. Murphy, Ph.D. thesis in preparation. 